\title{
Surface Science of Metal Oxides: Examining What Happens at the Atomic Scale ${ }^{+}$
}

\author{
Ulrike Diebold \\ Institute of Applied Physics, TU Wien, Wiedner Hauptstrasse 8-10/134, A-1040 Vienna, Austria; \\ diebold@iap.tuwien.ac.at \\ + Presented at the 4th International Conference nanoFIS 2020-Functional Integrated nano Systems, Graz, \\ Austria, 2-4 November 2020.
}

Published: 17 December 2020

\begin{abstract}
The atomic-scale phenomena at surfaces and interfaces influence, and often even dominate, the properties of materials and their functioning in nanoscale devices. This contribution discusses recent results of applying the surface science method, where systems are investigated under idealized conditions. Such experiments directly relate to first-principles calculations and provide insights into mechanisms and processes at a level that cannot be achieved in any other way. The review discusses recent developments with a main emphasis on metal oxides, a versatile and extremely useful class of materials.
\end{abstract}

Keywords: surface science; scanning probe microscopy; surface chemistry; chemical sensing; growth processes

\section{Introduction}

Scanning probe microscopy with atomic resolution provides a direct view at atomic and local electronic structure, and can even be used to follow molecular processes dynamically in real time. This works best under stringent control of the environment and the sample purity, i.e., under ultrahigh vacuum (UHV) conditions and using single crystals.

The surface chemistry of many materials, most prominently of metal oxides, is often defectdominated. Atomically-resolved scanning tunneling microscopy (STM) lends itself perfectly to investigate such point defects and directly shows the various types, including isolated oxygen vacancies, single adatoms, and cation deficiencies in the near-surface region [1]. Recent developments in UHV-based non-contact atomic force microscopy (ncAFM), where piezoelectric sensors are combined with the metal tips usually used in STM [2], appropriately functionalized with a single molecule [3], or negatively or positively charged atoms [4], have opened a new way of examining the atomic-scale structure and properties of such systems [5].

\section{A Case Study in Surface Chemistry: Adsorption of $\mathrm{O}_{2}$ on Semiconducting Metal Oxides}

Molecular $\mathrm{O}_{2}$ on semiconducting metal oxides shows a surprisingly rich surface chemistry with obvious ramifications in the chemical sensing and catalysis. With recent ncAFM measurements, we showed how the charge state of individual adsorbed molecules can be controlled [6], and how different adsorption configurations can be identified based on imaging combined with experimental and theoretical force-distance curves [7].

\section{Combing Surface Science and Pulsed Laser Deposition: An Unusual Approach}

The industry standard for growing complex metal oxides is pulsed laser deposition (PLD) under epitaxial control with reflection high-energy electron diffraction (RHEED). We will discuss 
experiments performed in a setup where we combine a state-of-the-art PLD chamber with a fully developed, UHV-based surface science chamber that includes STM with electron spectroscopies and diffraction [8]. Here, the growth can be followed pulse by pulse, which allows a disentanglement of the various parameters (sample temperature, laser fluence, gas pressure, and step density) that affect film morphology [8]. For ternary metal oxides such as $\mathrm{SrTiO}_{3}$, the surface structure is sensitively controlled by the surface stoichiometry. We have quantitively mapped out the phase diagram of $\mathrm{SrTiO}_{3}(110)$ [9]. This provides the basis to relate atomic-scale surface reconstruction to the surface composition, but also to reproducibly and reversibly adjust the surface structure on the same bulk material. This capability was used for devising a novel method to determine the cation flux in PLD with unprecedented precision [10], to understand the influence of surface structure on the evolving growth morphology [11], and to understand how the surface reconstructions control the incorporation of oxygen in model cathodes for solid oxide fuel cells [12].

Funding: This research was funded by the Austrian Science Fund, FWF, grant number Wittgenstein-Prize ( $Z$ 250-N27).

Conflicts of Interest: The author declares no conflict of interest.

\section{References}

1. Setvin, M.; Wagner, M.; Schmid, M.; Parkinson, G.S.; Diebold, U. Surface point defects on bulk oxides: atomically-resolved scanning probe microscopy. Chem. Soc. Rev. 2017, 46, 1772-1784.

2. Giessibl, F.J. The qPlus sensor, a powerful core for the atomic force microscope. Rev. Sci. Instrum. 2019, 90, 011101-60.

3. Gross, L.; Mohn, F.; Moll, N.; Liljeroth, P.; Meyer, G. The Chemical Structure of a Molecule Resolved by Atomic Force Microscopy. Science 2009, 325, 1110-1114.

4. Onoda, J.; Ondráček, M.; Jelínek, P.; Sugimoto, Y. Electronegativity determination of individual surface atoms by atomic force microscopy. Nat. Commun. 2017, 8, 15155.

5. Setvín, M.; Reticcioli, M.; Poelzleitner, F.; Hulva, J.; Schmid, M.; Boatner, L.A.; Cesare Franchini, U.D. Polarity compensation mechanisms on the perovskite surface $\mathrm{KTaO}_{3}(001)$. Science 2018, 359, 572-575.

6. Setvín, M.; Hulva, J.; Parkinson, G.S.; Schmid, M.; Diebold, U. Electron transfer between anatase $\mathrm{TiO}_{2}$ and an $\mathrm{O}_{2}$ molecule directly observed by atomic force microscopy. Proc. Natl. Acad. Sci. USA 2017, 114, E2556-E2562.

7. Sokolović, I.; Reticcioli, M; Čalkvosky, M; Wagner, M; Franchini, C; Schmid, M; Diebold, U; Setvín, ; Atomistic insights into the adsorption of molecular $\mathrm{O}_{2}$ on the rutile $\mathrm{TiO}_{2}(110)$ surface. Proc. Natl. Acad. Sci. USA 2020, 117, 14827-24837.

8. Gerhold, S.; Riva, M.; Yildiz, B.; Schmid, M.; Diebold, U. Adjusting island density and morphology of the $\mathrm{SrTiO}_{3}(110)-(4 \times 1)$ surface: Pulsed laser deposition combined with scanning tunneling microscopy. Surf. Sci. 2016, 651, 76-83.

9. Wang, Z.; Loon, A.; Subramanian, A.; Gerhold, S.; McDermott, E.; Enterkin, J.A.; Hiecke, M.; Russel, B.C.; Green, R.J.; Moewes, A.; et al. Transition from Reconstruction toward Thin Film on the (110) Surface of Strontium Titanate. Nano Lett. 2016, 16, 2407-2412.

10. Riva, M.; Franceschi, G.; Lu, Q.; Schmid, M.; Yildiz, B.; Diebold, U. Pushing the detection of cation nonstoichiometry to the limit. Phys. Rev. B 2019, 3, 043802.

11. Riva, M.; Franceschi, G.; Schmid, M.; Diebold, U. Epitaxial growth of complex oxide films: Role of surface reconstructions. Phys. Rev. Res. 2019, 1, 033059.

12. Riva, M.; Kubicek, M.; Hao, X.; Franceschi, G.; Gerhold, S.; Schmid, M.; Hutter, H.; Fleig, J.; Franchini, C.; Yildiz, B.; et al. Influence of surface atomic structure demonstrated on oxygen incorporation mechanism at a model perovskite oxide. Nat. Commun. 2018, 9, 916-919.

Publisher's Note: MDPI stays neutral with regard to jurisdictional claims in published maps and institutional affiliations. 